\title{
Metabolic disturbances induce malignant heart arrhythmias in rats
}

\author{
Liptak B ${ }^{1,2}$, Knezl V', Gasparova $Z^{1}$ \\ Institute of Experimental Pharmacology and Toxicology, Slovak Academy of Sciences, \\ Bratislava, Slovakia. liptaq.b@gmail.com
}

\section{ABSTRACT}

OBJECTIVES: Metabolic disturbances are considered to condition the occurrence of malignant heart arrhythmias and negatively influence the chances of a patient to survive. To test this assumption, a model of metabolic syndrome was selected in which rats were receiving a diet resembling that of the westernized population. BACKGROUND: Metabolic syndrome is a comorbidity of major cardiovascular risk factors (dyslipidemia, hypertension, impaired glucose tolerance or insulin resistance, diabetes mellitus, and obesity), all facilitating cardiovascular complications leading to morbidity and mortality of patients.

METHODS: Hearts were isolated and perfused according to Langendorff. Global ischemia was induced in the hearts and arrhythmia occurrence in reperfusion was monitored. All hearts were stimulated with the electrocardio-stimulator to test the electrical inducibility of heart arrhythmia.

RESULTS: Isolated hearts from rats with the metabolic syndrome were more susceptible to ventricular arrhythmias. The high-fat diet increased the occurrence of malignant heart arrhythmias in rats with metabolic syndrome to an even greater extent. All subjects with metabolic syndrome were sensitive to ventricular tachyarrhythmia with significantly decreased threshold to its induction in cardio-stimulation.

CONCLUSIONS: These results indicate that metabolic syndrome patients may be more sensitive to the occurrence of malignant heart arrhythmias following myocardial infarction or other heart diseases (Tab. 1, Fig. 2, Ref. 34). Text in PDF www.elis.sk.

KEY WORDS: dyslipidemia, ischemia-reperfusion, Langendorff, ventricular fibrillation, western-diet.

\section{Introduction}

Dyslipidemia, including cholesterol imbalance and hypertriacylglyceridemia, is a clinically important hallmark of atherosclerosis - an underlying cause of about $50 \%$ of all deaths in westernized societies (1). Dyslipidemia is characterized by increased triacylglycerols (TG), elevated low-density lipoprotein cholesterol (LDL) and very low-density lipoprotein cholesterol (VLDL), chylomicrons, and decreased high-density lipoprotein cholesterol (HDL), a case commonly observed in cardiovascular patients and patients with metabolic syndrome $(2,3)$. Thus the lipoprotein fraction and lipid profile are inevitable factors of metabolic syndrome criteria and risk factors for cardiovascular disease (4). Negative lifestyle adaptations are irrefutably linked to emergence of these health complications (5) leading to cardiovascular disease.

${ }^{1}$ Institute of Experimental Pharmacology and Toxicology, Slovak Academy of Sciences, Bratislava, Slovakia, and ${ }^{2}$ Department of Pharmacology, Jessenius Faculty of Medicine, Comenius University in Bratislava, BIOMED, Martin, Slovakia

Address for correspondence: B. Liptak, Institute of Experimental Pharmacology and Toxicology, Slovak Academy of Sciences, Dubravska cesta 9, SK-841 04 Bratislava, Slovakia Phone: +421.910119231

Acknowledgement: This study was financially supported by the VEGA grant No. 2/0054/15.
Chronic elevation of TG, LDL and VLDL cholesterol emerge as a consequence of unhealthy lifestyle (stress and reduced physical activity) and diet, characteristic for the way of life of a significant part of the western population (6). Cardiovascular mortality is extremely high. It represents overall mortality of approximately $47 \%$ in Europe, $33.7 \%$ in Americans and $30.8 \%$ in the USA (7, $8,9)$. A major cause of cardiovascular mortality are malignant heart arrhythmias. They represent a major clinical problem with unsatisfactory management. Ventricular fibrillation (VF) appears after myocardial infarction in about $20 \%$ cases with significant probability of their appearance within the first three hours after the incident $(10,11)$. Prognosis of survival is determined by many factors. Metabolic syndrome patients, patients with diabetes mellitus type II (DM), and obese individuals may have decreased chances of survival. In these patients, malignant heart arrhythmias may occur more frequently, with increased duration and severity. Ventricular and atrial arrhythmias may thus result in severe morbidity, coma and death. More than half of the patients with ischemic heart disease (IHD) died due to ventricular arrhythmias and ventricular tachyarrhythmias, which are the most common and most severe form of heart dysrhythmias occurring in $84 \%$ of patients with sudden cardiac death (12). The incidence of coronary heart disease is increased threefold in patients with metabolic syndrome, with markedly increased cardiovascular morbidity (13). Metabolic syndrome has been identified in $\sim 35 \%$ of patients in the USA (14) and ranges from $8 \%$ to $43 \%$ in men and $7 \%$ to 


\section{9-543}

$56 \%$ in women across the world $(15,16)$. Approximately $10 \%$ of the population in Europe has DM type II (17) and more than a half of obese patients (BMI $\geq 30)$ was identified with metabolic syndrome (18). In the Botnia study, $10 \%$ and $15 \%$ of subjects were identified with metabolic syndrome having normal glucose tolerance, $42 \%$ and $64 \%$ with impaired fasting glucose or impaired glucose tolerance and $78 \%$ and $84 \%$ of those with MD type II (13). In the USA, obesity is present in more than one-third of adults and its prevalence is $17 \%$ in children (19). Obesity in Europe ranges from $4.0 \%$ to $28.3 \%$ in men and $6.2 \%$ and 36.5 $\%$ in women (20). Hypertension has been identified in $31.1 \%$ of adults (21) with a respective $74.3 \%$ and $70.2 \%$ prevalence in men and women of 45-83 years of age (22). Elevated total cholesterol concentrations ( $\geq 190 \mathrm{mg} / \mathrm{dL}$ ) were found in $60.5 \%$ of women and $56.6 \%$ of men in the German population (23) and dyslipidemia occurs in $52.9 \%$ of adults in the USA (24) and $53.6 \%$ in China (25). All these above mentioned chronic diseases are established cardiovascular risk factors. Moreover, they are found to be interlinked and to promote the establishment of metabolic syndrome (26), a comorbidity of factors that increase cardiovascular events, morbidity and mortality $(27,28)$.

To test our predictions, a model of the metabolic syndrome (according to animal model described by Zicha et al (29) was applied and the hearts isolated from these animals were perfused according to Langendorff (see 30). To simulate the lifestyle of the western population, a high-fat diet was given to these laboratory animals (described in Kaprinay et al (31). Our hypotheses were as follows: 1) dyslipidemia accompanied with other metabolic disturbances of the metabolic syndrome will result in heavier ischemiareperfusion injury with an increase in malignant heart arrhythmias in comparison to healthy Wistar rats; 2) hypertriacylglycerolemic (HTG) rats receiving high-fat diet will have increased ischemiareperfusion injury and an increase in malignant heart arrhythmias to an even greater extent in comparison with HTG rats with standard rat chow (SD).

\section{Material and methods}

All procedures involving animals were performed in compliance with the Principles of Laboratory Animal Care issued by the Ethical Committee of the Institute of Experimental Pharmacology and Toxicology and the State Veterinary and Food Administration of Slovakia. Prague hereditary HTG rats $(n=20)$ and Wistar rats (n $=10$ ) were obtained from the breeding station Dobra Voda (Slovak Republic, reg. No. SK CH 24016). The animals were divided into three groups (each containing ten animals). The laboratory rats were then transferred into boxes. Each box contained five animals. After a one week lasting quarantine, the animals were kept in the housing room for 8 weeks. At the beginning of housing, the diet of one group $(n=10)$ of the HTG rats was exchanged for the high-fat diet (HFD) containing $7.5 \%$ lard and $1 \%$ cholesterol ad libitum (HTG-HFD). The other two groups, Wistar $(\mathrm{n}=10)$ and HTG (n $=10$ ) rats, received SD ad libitum. At the end of the experiment all rats were 18 weeks old with an average weight of about. 400 g. Metabolic syndrome developed in all HTG rats in both groups (standard diet and high-fat diet group). Detailed description of the molecular markers and blood samples is published in our mutual paper written by Kaprinay et al (31). In brief: All HTG rats in both groups (HTG-SD and HTG-HFD) had in comparison to control (W-SD) rats an increased triacylglycerol level $(4.5 \pm 0.3 \mathrm{mmol} / 1$ and $2.2 \pm 0.1 \mathrm{mmol} / 1$ vs $0.65 \pm 0.09 \mathrm{mmol} / \mathrm{l})$, decreased $\mathrm{HDL}$ cholesterol $(0.26 \pm 0.03 \mathrm{mmol} / \mathrm{l}$ and $0.35 \pm 0.05 \mathrm{mmol} / 1$ vs $0.68 \pm$ $0.01 \mathrm{mmol} / \mathrm{l})$, increased systolic blood pressure $(129.3 \pm 2.5 \mathrm{~mm}$ $\mathrm{Hg}$ and $128.7 \pm 2.5 \mathrm{~mm} \mathrm{Hg}$ vs $113.5 \pm 2.9 \mathrm{~mm} \mathrm{Hg})$, higher preprandial glucose $(5.1 \pm 0.13 \mathrm{mmol} / \mathrm{l}$ and $5.8 \pm 0.2 \mathrm{mmol} / \mathrm{l} \mathrm{vs} 3.96$ $\pm 0.1 \mathrm{mmol} / \mathrm{l})$, and impaired glucose tolerance $(9.0 \pm 0.3 \mathrm{mmol} / 1$ and $8.2 \pm 0.5 \mathrm{mmol} / \mathrm{l} \mathrm{vs} 3.96 \pm 0.09 \mathrm{mmol} / \mathrm{l})$. LDL cholesterol was significantly elevated only in the HTG-HFD group in comparison to the W-SD and HTG-SD groups $(1.54 \pm 0.02 \mathrm{mmol} / \mathrm{l}$ vs $0.22 \pm$ $0.02 \mathrm{mmol} / \mathrm{l}$ and $0.19 \pm 0.01 \mathrm{mmol} / \mathrm{l})$. Body weight of the HTG animals following standard diet and high-fat diet was not significantly increased in comparison to Wistar control group (362.4 \pm $1.5 \mathrm{~g}$ and $377.2 \pm 1.2 \mathrm{~g}$ vs $396.3 \pm 0.9 \mathrm{~g}$ ).

The hearts of all laboratory rats were isolated according to Langendorff. After the animals were transported into the laboratory, they were anesthetized with diethyl ether and immediately decapitated. The heart was rapidly dissected and cannulated in situ. The same researcher conducted all the isolations. After successful cannulation and initiation of the perfusion, all hearts were put immediately on the Langendorff apparatus for retrograde perfusion. The apparatus was set to constant pressure $(80 \mathrm{~mm} \mathrm{Hg})$. KrebsHenseleit bicarbonate perfusion solution was prepared as follows (in $\mathrm{mM}$ ): $\mathrm{NaCl}$ (118), $\mathrm{KCl}$ (4.7), $\mathrm{CaCl}_{2}$ (2.5), $\mathrm{NaH}_{2} \mathrm{PO}_{4}$ (1.18), $\mathrm{NaHCO}_{3}(25)$, and glucose (11.1) equilibrated with a gas mixture of $95 \% \mathrm{O}_{2}$ and $5 \% \mathrm{CO}_{2}$ at $37{ }^{\circ} \mathrm{C}$, pH 7.4. Electrocardiograph electrodes were put on the heart right atrium and left ventricle and a latex balloon was inserted into the left ventricle immediately after placing the heart on the apparatus. After insertion of the latex balloon, the volume of the balloon was filled with water and the tubular balloon holder was connected to the pressure transducer via a tube. Left ventricular end-diastolic pressure (LVEDP) was set to give an approximate power of $0.5 \mathrm{kPa}(\sim 10 \mathrm{~mm} \mathrm{Hg})$ before starting the measurements. Tesla electro-manometers were used to monitor the left ventricle pressure. The developed left ventricular pressure (LVDP), LVEDP, heart rate (HR), coronary flow (CF), $-\mathrm{dP} / \mathrm{dt}$ and $+\mathrm{dP} / \mathrm{dt}$ were subsequently monitored with the BioLabF software throughout the experiment. After the setting all hearts were kept stabilized for 15 minutes. After stabilization, 20 minutes lasting global ischemia followed. During the ischemia, all hearts were submerged in Krebs-Henseleit solution at $37^{\circ} \mathrm{C}$. The 20 -minute ischemia was followed by 20 -minute reperfusion of the heart. Then the stimulation electrodes were put on the myocardium and ascending stimulation followed with doses from $10 \mathrm{~mA}$ to 50 $\mathrm{mA}$. The goal was to induce sustained (2 minutes lasting) VF or ventricular tachycardia (VT). If sustained VF or VT developed during the reperfusion and persisted until the end of the reperfusion, the hearts were not conducted to cardio-stimulation. After the induction of sustained VF or VT ( $2 \mathrm{~min}), \mathrm{CF}$ was stopped and sinus rhythm recovery was monitored. During the reperfusion, the ventricular premature beats (VPB), bradycardia (B), VT and VF 
were monitored. Analytic software from the BioLabF was used to obtain the data. STATISTICA 10 software was used for the statistics. ANOVA Fisher LSD test was used for data comparison. All data are mean with \pm SEM. $* \mathrm{p}<0.05, * * \mathrm{p}<0.01, * * * \mathrm{p}<0.001$.

\section{Results}

Basic physiological features of the isolated hearts are shown in Table 1. Baseline values at the end of $15 \mathrm{~min}$ stabilization period were not statistically significantly different among groups. In reperfusion, however, a statistically significant increase in LVDP was recorded in the HTG-HFD group $(\mathrm{p}<0.036 \mathrm{~W}-\mathrm{SD}$ vs HTG-HFD and $p<0.008$ HTG-SD vs HTG-HFD). Coronary flow was higher in the HTG-SD and HTG-HFD group in comparison to W-SD ( $\mathrm{p}$ $<0.001$ and $\mathrm{p}<0.036) .+\mathrm{dP} / \mathrm{dt}$ was significantly increased in the HTG-HFD group compared to the HTG-SD group $(\mathrm{p}<0.009)$.
It is also noteworthy that a significant increase in LVEDP development was recorded for the HTG-SD group in comparison to W-SD $(\mathrm{p}<0.001)$.

At least two minutes lasting ventricular tachyarrhythmias occurring spontaneously during 20 minutes of reperfusion were observed in 3 out of 10 animals in the W-SD group, in 5 out of 10 animals in the HTG-SD group, and in 8 out of 10 animals in the HTG-HFD group. A mean number of 149.9 VPB (extrasystoles) developed in the W-SD group, 198.6 in the HTG-SD group, and 222 in the HTG-HFD group. A mean duration of $165.1 \mathrm{~s}$ lasting ventricular tachycardia developed in the W-SD group, $190.3 \mathrm{~s}$ in the HTG-SD, and $468.3 \mathrm{~s}$ in the HTG-HFD group. Only one animal developed VF in the W-SD group lasting 149.9 s. Five animals developed VF in the HTG-SD group with a mean duration of $127.8 \mathrm{~s}$. Four animals developed VF in the HTG-HFD group with a mean duration of $192.7 \mathrm{~s}$. Three animals developed bra-

Tab. 1. Baseline values of control Wistar rats (W-D), and HTG rats with the standard diet (HTG-SD) and high fat-diet (HTG-HFD).

\begin{tabular}{|c|c|c|c|c|c|c|}
\hline \multirow{2}{*}{$\begin{array}{l}\text { Period } \\
\text { Groups }\end{array}$} & \multicolumn{3}{|c|}{$15^{`}$ stabilisation } & \multicolumn{3}{|c|}{$20 `$ reperfusion } \\
\hline & W-SD & HTG-SD & HTG-HFD & W-SD & HTG-SD & HTG-HFD \\
\hline Heart rate (beats/min) & $272.36 \pm 14.49$ & $284.12 \pm 6.08$ & $260.1 \pm 15.38$ & $227 \pm 17.1$ & $246.85 \pm 13.73$ & $231.68 \pm 18.89$ \\
\hline Left ventricular developed pressure (LVDP) $(k P a)$ & $16 \pm 0.51$ & $15.95 \pm 1.05$ & $16.56 \pm 0.66$ & $4.05 \pm 0.89$ & $3.51 \pm 0.5$ & $6.17 \pm 0.48 * / x x$ \\
\hline Left ventricular end-diastolic pressure (LVEDP) $(\mathrm{kPa})$ & $0.31 \pm 0.13$ & $0.65 \pm 0.25$ & $0.66 \pm 0.28$ & $5.11 \pm 0.44$ & $5.4 \pm 0.39$ & $4.51 \pm 0.89$ \\
\hline Coronary flow $(\mathrm{ml} / \mathrm{min})$ & $15.53 \pm 0.51$ & $17.41 \pm 0.64$ & $17.02 \pm 1.05$ & $11.66 \pm 0.49$ & $14.89 \pm 0.69^{* *}$ & $13.85 \pm 0.82^{*}$ \\
\hline$-\mathrm{dP} / \mathrm{dt}(k P a / s)$ & $270.34 \pm 9.19$ & $294.97 \pm 24.65$ & $295.53 \pm 16.96$ & $59.49 \pm 17.07$ & $60.4 \pm 9.31$ & $98.19 \pm 12.09$ \\
\hline$+\mathrm{dP} / \mathrm{dt}(\mathrm{kPa} / \mathrm{s})$ & $385.91 \pm 17.11$ & $372.61 \pm 35.28$ & $395.09 \pm 27.12$ & $85.75 \pm 21.68$ & $64.46 \pm 10.82$ & $133.03 \pm 16.64^{x}$ \\
\hline
\end{tabular}

Values are presented as means \pm SEM. $* \mathrm{p}<0.05, * * \mathrm{p}<0.01, * * * \mathrm{p}<0.001$ comparing W-SD to HTG-SD and HTG-HFD, and ${ }^{\mathrm{x}} \mathrm{p}<0.05,{ }^{\mathrm{xx}} \mathrm{p}<0.01,{ }^{\mathrm{xxx}} \mathrm{p}<0.001$ comparing HTG-SD to HTG-HFD in 20' reperfusion. W-SD - Wistar rats-standard diet, HTG-SD -hypertriacylglycerolemic rats-standard diet, HTG-HFD - hypertriacylglycerolemic rats - high-fat diet
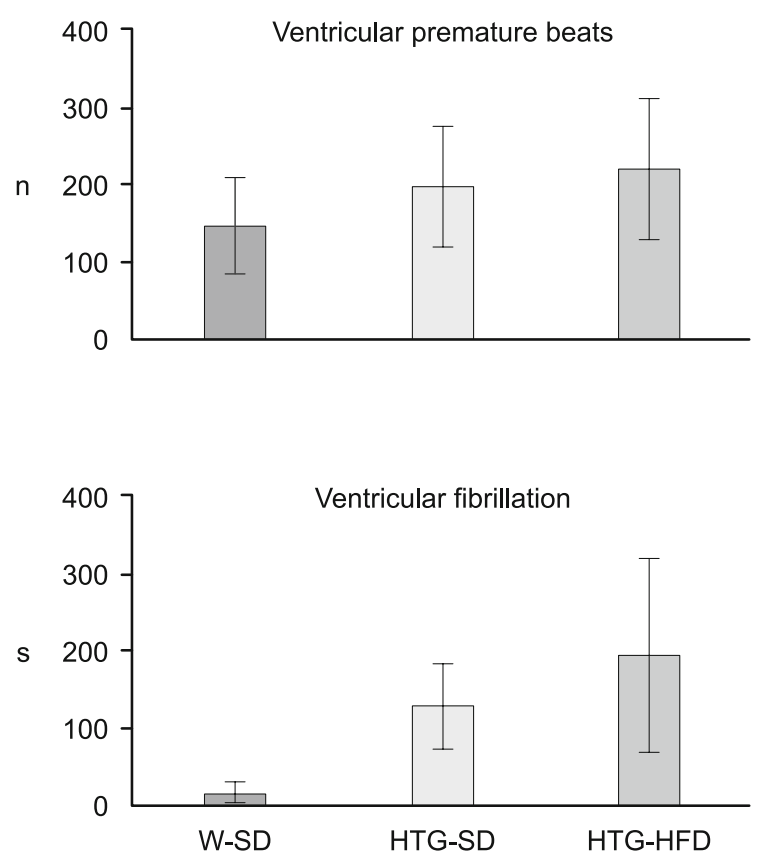
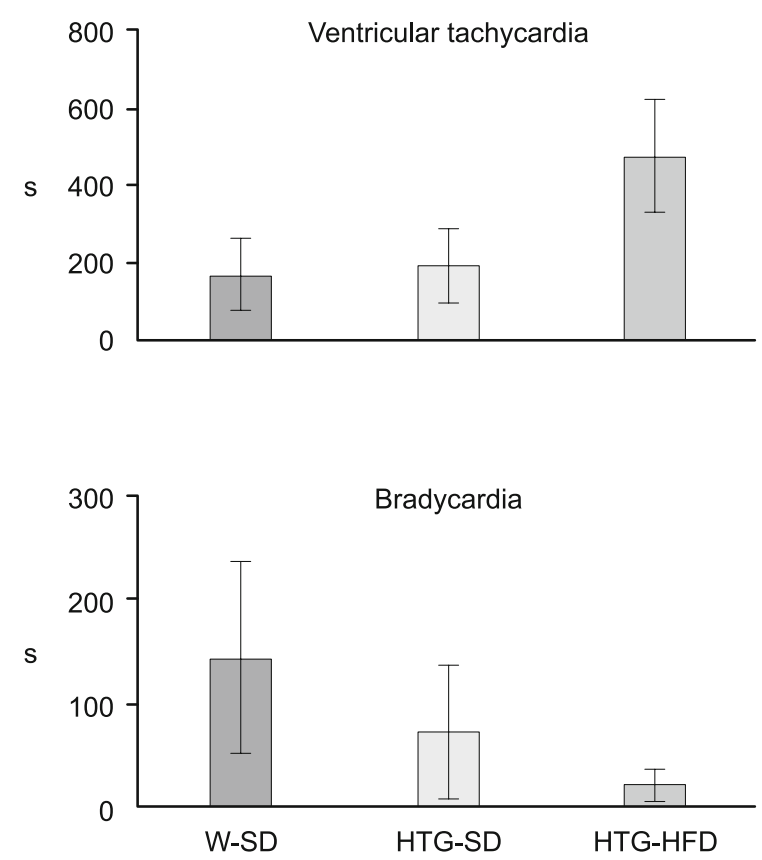

Fig. 1. Prevalence of ventricular premature beats (VPB), ventricular tachycardia (VT), ventricular fibrillation (VF) and bradycardia (B) in 20 min reperfusion. Values are presented as means \pm SEM. No significant differences were statistically identified. W-SD - Wistar rats-standard diet, HTG-SD - hypertriacylglycerolemic rats-standard diet, HTG-HFD - hypertriacylglycerolemic rats - high-fat diet. 


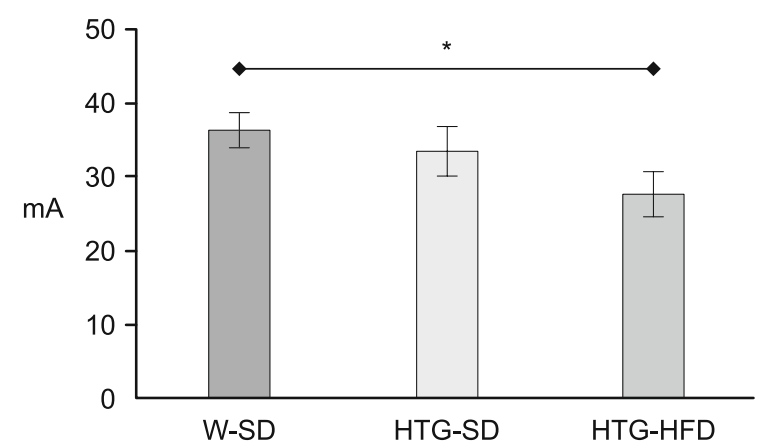

Fig. 2. Sensitivity to tachyarrhythmia inducement during cardiostimulation. Values are presented as means \pm SEM. * $p<0.05$. W-SD vs HTG-HFD p < 0.044. W-SD - Wistar rats-standard diet, HTG-SD -hypertriacylglycerolemic rats-standard diet, HTG-HFD - hypertriacylglycerolemic rats - high-fat diet.

dycardia in the W-SD group, while only two animals developed bradycardia in the HTG-SD and HTG-HFD groups. A summary of arrhythmias is presented in Figure 1.

During stimulation, 6 out of 10 animals developed 2 minutes lasting sustained tachyarrhythmias in the W-SD group, 7 out of 9 (one animal was not stimulated due to persisting arrhythmia developed at the end of reperfusion) in the HTG-SD group, and 6 out of 6 (four animals developed sustained arrhythmia at the end of reperfusion and were not stimulated) in the HTG-HFD group. Isolated hearts of the $\mathrm{W}$-SD groups were more resistant to inducement of ventricular arrhythmia than HTG-HFD $(\mathrm{p}<0.044 \mathrm{~W}-\mathrm{SD}$ vs HTG-HFD) (Fig. 2). Sinus rhythm recovery was not statistically significantly different among the groups.

\section{Discussion}

Current predominant lifestyle has led to the occurrence of many chronic diseases, and of cardiovascular diseases in particular. Ventricular malignant arrhythmias contribute significantly to morbidity and mortality of these cardiovascular patients. A recent study of Albarado-Ibaňez et al (32) demonstrated that metabolic syndrome remodels electrical activity of the sinoatrial node and produces arrhythmias in rats. Our study shows development of malignant heart arrhythmia in isolated hearts on the Langendorff apparatus. Metabolic disorders expressed in this metabolic syndrome model in both dietary groups of the HTG rats, in comparison to the Wistar control group, were an increase in VPB count, duration and frequency of VT and VF in an ascending order W-SD $<$ HTG-SD $<$ HTG-HFD. Although the statistical analysis failed to give statistical significance because of a considerable variance in the duration of arrhythmogenic events, deterioration of isolated HTG rat hearts was evident from the recordings and obtained data. Summarization of total duration of all VT or VF episodes of all rats indicates a clear progression of malignant heart arrhythmia in HTG-SD rats, with an even higher degree in HTG-HFD rats. Decrease in bradyarrhythmia observed in HTG rats is explained through a shift of the heart to a more severe reaction and imbal- ance resulting mainly as VT and VF. Decreased cardiac susceptibility to arrhythmia induction in cardio-stimulation supports our observations in favour of the pro-arrhythmic effect of metabolic syndrome comorbidity identified in HTG-HFD rats. HFD without any doubt worsened the outcomes of hearts isolated from the HTG-HFD group compared with the HTG-SD and W-SD groups. The HFD consisting of standard pellet enriched with $7.5 \%$ lard and $1 \%$ cholesterol contributed significantly to the genesis of heart arrhythmia.

The Prague hereditary hypertriacylglycerolemic rats are not the commonest used model of the metabolic syndrome but they resemble selected symptoms very well (29). Commonly used rat models are spontaneous hypertensive rats and Sprague Dawley rats, both strains fed with high-sugar diet (33). Although high-fat diet models and high-sucrose models have different effects on the metabolism, both facilitate metabolic disorders (34). Spontaneous HTG rats and HTG rats fed high-fat diet both resemble signs of the metabolic syndrome (31). High-fat diet however affects the heart sinus rhythm to a higher extent, leading to a reduced fibrillation threshold and a more frequent occurrence in ventricular fibrillation. Sinus rhythm disturbances occurring in the metabolic syndrome (32) may thus lead to heart arrhythmias, manifested as ventricular tachycardia and fibrillation, as observed in our experiment. Based on observations conducted on isolated hearts from animals with metabolic syndrome, the authors stress the importance to translate these findings to clinical practice and to take in mind the increased probability of VF development in patients with metabolic syndrome. They would also suggest to prescribe anti-arrhythmic drugs as prevention of cardiac morbidity and mortality.

\section{Learnings points}

The rat subjects expressing the metabolic syndrome developed malignant heart arrhythmias to a greater extent in comparison to healthy controls.

\section{References}

1. Lusis JA. Atherosclerosis. Nature 2000; 407: 233-241.

2. Laufs U, Bohm M, Weingartner O, Werner C, Custodis F, Poss J. Cardiovascular disease and dyslipidemia: beyond LDL. Curr Pharm Design 2011; 17: 861-870.

3. Grundy SM. Metabolic syndrome: connecting and reconciling cardiovascular and diabetes worlds. J Am Coll Cardiol 2006; 47: 1093-1100.

4. Alberti KGMM, Eckel RH, Grundy SM, et al. Harmonizing the metabolic syndrome. A joint interim statement of the International Diabetes Federation Task Force on Epidemiology and Prevention; National Heart, Lung and Blood Institute; American Heart Association; World Heart Federation; International Atherosclerosis Society; and International Association for the Study of Obesity. Circulation 2009; 120: 1640-1645.

5. Warburton DER, Nicol CW, Bredin SSD. Heath benefits of physical activity: the evidence. CMAJ 2006; 147: 801-809.

6. Ahmed MH, Blaha JM, Nasir K, Rivera JJ, Blumenthal SR. Effects of physical activity on cardiovascular disease. Am J Cardiol 2012; 109: 288-295. 
7. Nichols M, Townsend N, Luengo-Fernandez R, Leal J, Gray A, Scarborough P, Rayner M (Eds.). European cardiovascular disease statistics 2012. Brussels, Sophia Antipolis: European Heart Network, European Society of Cardiology, 2012: 1-125.

8. de Fatima Marinho de Souza M., Gawriszewski VP, Orduňez P, Sanhueza A, Espinal MA. Cardiovascular disease mortality in the Americas: current trends and disparities. Heart 2012; 98: 1207-1212.

9. Mozaffarian D, Benjamin EJ, Go SA, et al. Heart disease and stroke statistics - 2016 update. A report from the American Heart Association. Circulation 2016; 133: e38-e360.

10. Campbell FWR, Murray A, Julian GD. Ventricular arrhythmias in first 12 hours of acute myocardial infarction. Natural history study. Brit Heart J 1981; 46: 351-357.

11. O'Doherty M, Tailer ID, Quinn E, Vincent R, Chamberlain AD. Five hundred patients with myocardial infarction monitored within one hour of symptoms. Brit Med J 1983; 286: 1405-1408.

12. Bayés de Luna A, Coumel P, Leclercq JF. Ambulatory sudden cardiac death: Mechanisms of production of fatal arrhythmia on the basis of data from 157 cases. Am Hearth J 1989; 117: 151-159.

13. Isomaa B, Almgren $\mathbf{P}$, Tuomi $\mathbf{T}$, et al. Cardiovascular morbidity and mortality with the metabolic syndrome. Diabetes Care 2001; 24: 683-689.

14. Aguilar M, Bhuket T, Torres S, Liu B, Wong RJ. Prevalence of the Metabolic Syndrome in the United States, 2003-2012. JAMA 2015; 313: 1973-1974.

15. Cameron AJ, Shaw JE, Zimmet PZ. The metabolic syndrome: prevalence in worldwide population. Endocrin Metab Clin 2004; 33: 351-375.

16. Kaur J. A comprehensive review on metabolic syndrome. Cardiol Res Pract 2014; 2014: 973162.

17. Tamayo T, Rosenbauer J, Wild SH, et al. Diabetes in Europe: an update. Diabetes Res Clin Pr 2014; 103: 206-217.

18. van Vliet-Ostaptchouk JV, Nuotio M-L, Slagter SN, et al. The prevalence of metabolic syndrome and metabolically healthy obesity in Europe: a collaborative analysis of ten large cohort studies. BMC Endocrine Disorders 2014; 14: 9.

19. Ogden CL, Caroll MD, Kit BK, Flegal KM. Prevalence of obesity in the United states, 2009-2010. NCHS Data Brief 2012; 82: 1-8.

20. Berghöfer A, Pischon T, Reinhold T, Apovian CM, Sharma AM, Willich SN. Obesity prevalence from a European perspective: a systematic review. BMC Public Health 2008; 8: 200.

21. Mills KT, Bundy JD, Kelly TN, et al. Global disparities of hypertension prevalence and control. A systematic analysis of population-based studies from 90 countries. Circulation 2016; 134: 441-450.
22. Lacruz ME, Kluttig A, Hartwig S, et al. Prevalence and incidence of hypertension in the general adult population. Medicine (Baltimore) 2015; 94: e952.

23. Scheidt-Nave C, Du Y, Knopf H, et al. Prevalence of dyslipidemia among adults in Germany. Results of the German Health Interview and Examination Survey for Adults (DEGS1). Bundesgesundheitsbl 2013; 56: 661-667.

24. Toth PP, Potter D, Ming EE. Prevalence of lipid abnormalities in the United States: the national health and nutrition examination survey 2003-2006. J Clin Lipidol 2012; 6: 325-330.

25. Gu D, Gupta A, Muntner P, Hu S, et al. Prevalence of cardiovascular disease risk factor clustering among the adult population of China: results from the International Collaborative Study of Cardiovascular disease in Asia (InterAsia). Circulation 2005; 112: 658-665.

26. Huang PL. A comprehensive definition for metabolic syndrome. Dis Model Mech 2009; 2: 231-237.

27. Roots CJ, Quax PHA, Jukema JW. Cardiovascular metabolic syndrome: mediators involved in the pathophysiology from obesity to coronary heart disease. Biomark Med 2012; 6: 35-52.

28. Grundy SM. Metabolic syndrome: a multiplex cardiovascular risk factor. J Clin Endocr Metab 2007; 92: 399-404.

29. Zicha J, Pecháňová O, Čačányiová $\mathrm{S}$, et al. Hereditary hypertriglyceridemic rat: a suitable model of cardiovascular disease and metabolic syndrome. Physiol Res 2006; 55 (suppl. 1): S49-S63.

30. Skrzypiec-Spring M, Grotthus B, Szeląg A, Schulz R. Isolated heart perfusion according to Langendorff - still viable in the new millennium. J Pharmacol Toxicol 2007; 55: 113-126.

31. Kaprinay B, Lipták B, Slovák L, Švík K, Sotníková R, Gáspárová Z. Hypertriglyceridemic rats fed high fat diet as a model of metabolic syndrome. Physiol Res 2016; 65 (Suppl. 4): S515-S518.

32. Albarado-Ibaňez A, Avelino-Cruz JE, Velasco M, Torres-Jácome J, Hiriart M. Metabolic syndrome remodels electrical activity of the sinoatrial node and produces arrhythmias in rats. PLoS ONE 2013; 8: e76534.

33. Oron-Herman M, Kamari Y, Grossman E, et al. Metabolic syndrome: comparison of the two commonly used animal models. Am J Hypertens 2008; 21: 1018-1022.

34. Sumiyoshi M, Sakanaka M, Kimura Y. Chronic intake of high-fat and high-sucrose diet differentially affects glucose intolerance in mice. $\mathrm{J}$ Nutr 2006; 136: 582-587. 